\title{
ESTUDOS LITERÁRIOS
}




\title{
POÉTIQUE DES MARGES
}

\author{
Richard-Laurent Barnett*
}

\section{Les marges comme "écriture"}

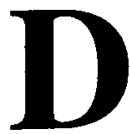

epuis près de deux siècles, la contestation du "centre" du système littéraire au profit de ses "marges" est devenue le plus répandu, le plus coriace des lieux communs. Chaque esthétique ou idéologie part en effet à l'assaut d'une citadelle jugée obsolète et liberticide pour la remplacer par une nouvelle conception de la littérature, elle aussi rapidement vouée au déclassement. Au nom des exigences inaliénables du moi, les romantiques et leurs successeurs, de nos jours encore bien vivants, s'en prennent aux divers avatars de l'académisme: de ce combat, la lutte contre l'alexandrin est le symptôme le plus manifeste'. Invoquant

* Marygrove College USA

1 Pour un aperçu de l'effacement du vers classique, voir Jacques Roubaud, La Vieillesse d'Alexandre (Paris: Maspero, 1978). Pour une analyse de la résistance de ce même vers, voir Didier Coste, "Le retour d'Alexandre", Formules, 1 (1997). Pour plus de détails sur les écritures modernes qui s'opposent au romantisme sans pour autant renouer avec l'alexandrin, voir Jan Baetens, $L$ 'Éthique de la contrainte (Louvain: Peeters, 1995). 
plutôt le culte du nouveau à tout prix, les modernités historiques, qui s'échelonnent de Baudelaire aux avant-gardes anciennes ou récentes ${ }^{2}$, livrent force batailles contre les principes crus stériles de leurs prédécesseurs: les mille et une facettes de la poétique de l'écart, laquelle d'ailleurs poursuit et parachève l'esprit romantique, témoignent saillamment de cette recherche. Nourris d'égalitarisme et prônant, avec toutefois un malaise accru ${ }^{3}$, les valeurs du multiculturalisme, les contemporains post-modernes s'attaquent, et non sans équivoque, à l'idéal moderne du rare et de l'exceptionnel: s'abstenant de faire le départ entre Tintin et Shakespeare, entre Mozart et Madonna ou encore entre le discours publicitaire et les langages de l'art, ils projettent au coeur du système les lois jusqu'ici moins côtées du métissage et de l'impureté, du populaire et du facile.

Quel que soit l'enjeu fondamental des mouvements en question renouvellement, destruction, restauration - le désir de placer au centre du système littéraire ce qui se voyait auparavant confiné à ses marges, représente une véritable invariante <<historique〉>. Le désir stratégique d'occuper la marge dans l'espoir d'engranger de la sorte un maximum de capital symbolique, et, corollairement, la crainte obsessionnelle d'être confondu avec les positions d'un centre périmé, récusé, détesté, voire tout simplement ignoré, sont là pour prouver que l'état marginal reste aujourd'hui, du moins dans les divers domaines de l'art, le plus apprécié, le plus prestigieux, bref le plus distingué qui soit. L'essor actuel des "discours minoritaires", même s'il pose autant de questions sociologiques que textuelles ou exégétiques proprement dites, n'est pas seulement l'effet d'une tribalisation de la parole et de l'éclatement du champ discursif qui en résulte: dans la mesure où ces "langages communautaires" réclament pour tout le monde le droit de parler et de chanter comme il entend, ils vont aussi dans le sens de cette tendance "lourde", inscrite dans la longue durée, qui assimile le progrès au rejet d'un discours dominant.

Au-delà ou en-deça des a prioris et convictions de tout un chacun, le choix des marges constitue, en termes barthésiens, une seule et même "écriture" 4 , un seul et même engagement historique en faveur d'une certaine façon d'inscrire sa parole face au public et face à l'histoire. C'est le règne sans partage du goût de la marge, ou du dégoût du centre; c'est aussi la stratégie sans faille de l' "ôte-toi de là que je m'y mette". C'est enfin la domination d'un type de parole où les qualités du sujet

2 Pour une analyse fouillée des enjeux et problèmes du projet moderne, cf. Antoine Compagnon, Les Cinq paradoxes de la modernité (Paris: Seuil, 1990).

3 Voir la polémique lancée par Stanley Fish dans "Boutique Multiculturalism", Critical Inquiry, 23: 2 (Winter 1997): 378-395.

4 On se réfère ici à Le Degré zéro de l'écriture (Paris: Seuil, coll. Points, 1972, plus particulièrement à la triade langue/style/écriture. 
prévalent partout sur l'achèvement de son énoncé. Toutes proportions gardées, le tableau suivant que brosse Régis Debray du monde des arts s'applique non moins exactement à l'univers des lettres:

Constante médiologique: moins l'image est médiumnique, plus elle se fait médiatique. De façon générale, moins un art transmet, plus il "communique". [. . .] Plus une oeuvre symbolise, plus l'artiste peut s'absenter de la scène. Moins l'oeuvre envoûte en revanche, plus la personne de l'artiste doit nous faire frissonner $[\ldots]^{5}$

Toute la question est cependant de savoir si la marginalité si gaiement assumée, l'exception si fièrement revendiquée, la rébellion si instinctivement recherchée, fonctionnent encore réellement comme des démarches hostiles à la pensée dominante ou si, en revanche (et ce sera le point de vue défendu dans ces pages), le parti pris inconditionnel de la marginalité, chacun faisant ce qu'il a bien envie de faire sans nul souci de rigueur formelle, ne représente pas plutôt le désir le plus confortable, le plus facile, en un mot le plus paresseux de tous face aux possibles engagements scripturaux. La réalité ne laisse subsister aucun doute à ce sujet, comme en témoignent bien les évolutions de l'enseignement: l'école n'est plus le garant de la culture littéraire légitime; ce que l'on impose dans les lycées, c'est le dérèglement des sens surréaliste, la métaphore débridée, le vers-librisme intransigeant et non plus les rigueurs de l'ancienne prosodie et versification; l'absence de règles est devenue pour l'écrivain en herbe quelque chose d'entièrement "naturel", c'està-dire un impératif social totalement intériorisé (daignerait-on s'en souvenir, la thèse de Borges que le vers libre est autrement plus difficile que le vers traditionnel ${ }^{6}$, ferait aujourd'hui bien sourire).

Or, s'il est vrai que les hiérarchies contemporaines voient pulluler en leur centre un grand nombre de discours pseudomarginaux, il convient évidemment de s'interroger sur les démarches qui pourraient constituer encore, sans pour autant toujours se désigner comme telles, les vraies marginalités. Le propos de cet article est de faire le tour des plus importants de ces discours “vraiment” marginaux, que l'on associera moins à des oeuvres

5 Vie et mort de l'image. Une histoire du regard en Occident. (Paris: Gallimard, coll. Folio, 1994), 86.

6 Les idées de Borges sur le vers libre sont reprises dans sa conférence sur le roman policier qui termine le volume collectif Autopsies du roman policier, ouvrage collectif dirigé par Uri Eizenzwecig (Paris: UGE, coll. 10/18, 1983). 
ou à des auteurs qu'à un ensemble complexe de choix, positifs ou négatifs, voulus ou subis, interdisant à ces travaux d'occuper le centre d'un système littéraire de plus en plus dominé par l'idéologie du moi-écrivant et de plus en plus indifférent aux valeurs et qualités, aux questions et aux impasses du texte même.

\section{L'illisible comme force irrécupérable}

S'il existe aujourd'hui un grand nombre de discours pseudomarginaux, autoproclamés ou non, les vrais marginaux sont sans doute ceux qui, pour diverses raisons, ne peuvent en aucune façon se faire admettre par le centre et qui cherchent même à s'en faire chasser comme impraticables ou infréquentables. De ce point de vue, rappelons-le, leur situation se sépare assez nettement des "discours des minorités" en vogue, où la reconnaissance par autrui - reconnaissance autant sinon plus sociale que littéraire du reste - constitue l'horizon ou la butée de l'écriture.

De toutes les écritures tenues à l'écart, celle de l'illisible (à mettre au pluriel, bien sûr) représente sans conteste l'avatar le moins ambigu. Cette catégorie a, de plus, l'avantage indéniable d'avoir trouvé en Christian Prigent, fondateur de la revue $\mathrm{TXT}^{7}$ un défenseur et un théoricien hors pair. Si le tir groupé de Ceux qui meredRent ${ }^{8}$ et $d^{\prime}$ Une erreur de la nature ${ }^{9}$, ses deux recueils consacrés à la question de l'illisible, n'a pas eu pour effet d'augmenter significativement l'audience des textes réputés illisibles, il est hors de doute que depuis les charges de Prigent contre la littérature fast-food de notre époque post-moderne, la catégorie d'illisible a pu acquérir, au moins dans les discussions théoriques, un vrai droit de cité, surtout auprès de ceux que n'inquiète aucune velléité de ne jamais à lire un volume de création de Prigent lui-même.

Car il convient d'y insister: les thèses sur l'équation radicale de la chose littéraire et de l'illisible ont rencontré un succès proportionnellement inverse à celui des textes récents dont Prigent s'est fait l'apôtre. Bien entendu pareille conjoncture: qui reflète un état de fait, engendre aussi des effets un rien pervers: elle confirme - faut-il le souligner - le clivage brutal et regrettable de la théorie et de la pratique, comme s'il était devenu possible de discuter de l'illisible sans qu'il faille passer par l'expérience des textes mêmes, là où Prigent fustige justement la subordination de l'acte d'écrire aux seuls en 1994.

7 La meilleure introduction au travail de cette revue est l'Anthologie TXT publiée chez Bourgois

8 Paris, P.O.L., 1991.

9 Paris, P.O.L., 1996 
programmes ou préoccupations théoriques, pour insister sur la valeur irremplaçable de l'excès du vécu par rapport aux limites de la raison théorique ${ }^{10}$.

Il y a d'autres problèmes encore, à commencer par la valorisation a priori de l'illisible quel qu'il soit, pourvu qu'il agace et exaspère les capacités de résistance du lecteur. Une telle démarche (faire illisible pour épater le bourgeois?) a sans doute le droit de faire trop vite l'économie des calculs stratégiques sans lesquels toute pratique de l'illisible serait vouée à l'inefficace (surtout aujourd'hui, à un moment historique où l'illisible n'est plus capable de choquer et où il est donc facile de l'ignorer purement et simplement). L'illisible est-il vraiment intéressant en soi? Ou l'est-il dans la mesure où il est lu, et lu d'une certaine manière?

De la même façon, si l'examen de l'illisible qu'on trouve chez Prigent distingue dans cette catégorie un certain nombre de sous-types, il y a une mise en evidence un peu trop systématique d'un genre très singulier d'illisible, celui de l'excès mis en oeuvre dans les textes inspirés d'Artaud et de Bataille, c'est-à-dire mais ce constat de militantisme ne signifie nullement un reproche - dans les écrits de Christian Prigent lui-même. Il est pourtant bien d'autres formes d'illisible et Une erreur de la nature permet fort bien d'en fait le tour:

Mais illisible en quoi, au fait?

Résumons:

(2) Trop cultivé, savant, cuistre (empêtré d" "allusions").

(3) Rompant les codes habituels de lecture (il faudrait lire en même temps - au prix de quel strabisme, de quel grand écart mental? - la page de droite et la page de gauche).

(4) Défaisant l'homogénéité en déplaçant plusieurs langues dans l'unique langue. (O. C., 23-24)

Cependant le même livre avère aussi les omissions intrigantes, dues au fait, suppose-t-on, que certaines oeuvres illisibles n'ont pas la moindre connivence thématique avec l'excès recherché par Prigent: les labyrinthes syntaxiques de Michel Falempin",

10 A cet égard, la comparaison - tout à fait justifiée - avec l’intervention théorique de Jean Ricardou dans le champ néo-romanesque des années 60 et 70 , ne manque pas d'être éclairante. Plus que dans le cas de Prigent, où le retour sur les oeuvres n'est pas toujours sûr, les travaux de Ricardou ont en effet vraiment servi, du moins à une certaine époque, de courroie de tranmission entre le lectorat et une production devenue, grâce à lưi, un peu moins illisible.

$11 \mathrm{Cf}$. par exemple Une apparence de vie (Paris: Ivréa, 1995). Pour une présentation de l'esthétique falempinienne, voir l'article de J. Baetens, "Vérités du détour", dans Formules, No. 1, 1997. 
par exemple, ou encore les mille et une facettes de la cryptographie oulipienne qu'on trouve dans l'oeuvre récente en prose de Jacques Roubaud ${ }^{22}$, entre autres.

Une approche plus différenciée, plus éclectique peut-être, qui accepte également de faire le départ entre les notions d'illisible d'un côté et l'illecturable de l'autre, paraît en ce sens plus apte à palier certaines impasses ${ }^{13}$. Il est en effet utile de ne pas confondre l'aspect psychologique et la dimension cognitive de la lecture: dans le premier cas, on opposera le lisible (qui désigne ce qu'il est agréable ou intéressant de lire) à l'illisible (qui désigne ce qu'on n'a pas envie de lire); dans le second cas, on opposera le lecturable (qui désigne les mécanismes du texte susceptibles d'être compris par le lecteur sans apport extérieur) à l'illecturable (qui désigne les mécanismes du texte qui ne peuvent pas être compris sans précisions ou aide externes). Les questions de stratégie susdites sont éclairées judicieusement par ces dichotomies à première sousdites vue en rien simplistes. Sans nul apport de lecturable, par exemple, il ne sera en effet jamais possible de discuter de façon un peu objective des leçons de l'illisible ou de l'illecturable (tant qu'on ne comprend pas ce qui est en jeu, la portée exacte du dérège element échappera forcément à l'attention et partant à toute appréciation critique). Corollairement, le jeu de l'illisible et du lecturable étant de nature profondément dialectique, le manque d'intérêt de certains textes peut être micux saisi à la lumière de cette tension: qu'a-t-on à faire par exemple d'un illisible dont le principe de (dé)composition saute si clairement aux yeux qu'on n'a guère envie de trop se pencher sur l'application mécanique d'un procédé devenu scie (le peu d'intérêt suscité par le texte ne provient alors pas du fait qu'on n'y comprend rien, mais du fait qu'on n'a que trop bien compris et qu'on n'a guère envie d'en savoir davantage)?

Enfin, l'accent répété mis sur le sujet, même dans la version hyperboliquement contestée qu'est le corps carnavalesque, travaillé et traversé par les pulsions les moins contrôlables de l'organisme, risque paradoxalement, non pas de flatter quelque doxa contemporaine, mais de rencontrer trop facilement l'exaltation finalement bien admise du moi de l'artiste. Danger de récupération moins totalement imaginaire que ne le pensent les jusqu'auboutistes de l'illisible.

12 Cf. surtout son cycle "autobiographique": Le grand incendie de Londres (Paris, Seuil, 1989), La boucle (id., 1993) et Mathématique (id., 1996).

13 Le concept de lecturable a été introduit par Benoît Peeters dans son étude Les Bijotux ravis, Bruxelles, éd. Magic Strip, 1983, et s'est vu notablement développé par Jean Ricardou dans son feuilleton théorique "Eléments de textique", publiés entre 1988 et 1991 dans la revue Conséquences. Dans une autre optique, à la fois tangentielle et pourtant différemment formulée, voir Richard-Laurent Barnett, "Transgressing Boundaries: The Poetics of Marginalisation", L'Esprit Créateur, No. 38, 1, 1998, "Poétique et marginalité", Seventeenth-Century French Studies (1998), Herméneutique et indicible (Paris: Seuil, à paraître). 


\section{L'excès et la règle}

Autant la position des tenants de l'illisible est dénuée de toute ambigüité, en tout cas du point de vue de la tension entre centralité et marginalité, autant la démarche des auteurs et des textes qui viennent de se rassembler sous la bannière de l'hyperconstruction peut prêter à confusion, du moins si on se limite à un examen du dehors et à très grande distance. Faisant front à la glorification romantique du sujet comme à la dépréciation aujourd'hui dominante des rigueurs de la forme, la revue Formules ${ }^{14}$, où cette tendance s'exprime le plus ouvertement, cherche à concilier deux exigences de nos jours tenues pour contradictoires: le respect des jeux formels (qui sont préférés à la hantise de l'expression de soi et la destruction iconoclaste des formes traditionnelles que l'on voit si répandues), puis le goût de l'expérience et la quête toute moderne d'un certain inouï qui n'est plus situé uniquement du côté de l'invention de techniques ou de procédés inédits, mais que l'on promet aussi dans l'emploi plus radical de formes déjà répertoriées et dont le grand lipogramme de Perec, La disparition ${ }^{15}$, fournit un exemple parlant.

Au niveau des exemples produits ou commentés, la démarche de Formules ne penche guère en faveur de quelque marginalité que ce soit (ce n'est pas un hasard si les grandes figures constamment invoquées se nomment Poe ou Mallarmé, Valéry ou Roussel, Perec, Queneau). Cependant les positions théoriques du groupe sont, quant à elles, beaucoup moins faciles à interpréter en termes de centralité ou de marginalité. La défense des formes fixes (le sonnet, la sextine, le vers holorime, etc.) ou l'évitement de toute la dérive inconoclaste sépare l'hyperconstruction des mouvements d'avant-garde mieux connus, si bien que pour des lecteurs pressés les convictions formalistes du groupe sont dangereusement proches de l'académisme. Dans cette perspective, il n'est pas absurde de croire que l'effort des hyperconstructeurs les porterait à vouloir restaurer ou réinvestir, certes de façon moins élitiste qu'avant, le centre de l'ancien système, centre abandonné depuis l'effondrement, vers les années de la "crise du vers", des contraintes pesant sur l'écriture poétique. Cependant la critique féroce des sous-entendus humanistes et romantiques des écritures contemporaines, toutes tendances confondues, fait que ces auteurs et ces textes composent un mouvement autrement plus marginal que les discours pseudomarginaux qui tiennent aujourd'hui le haut du pavé. La défense

14 Formules (revue annuelle): c/o Barnardo Schiavetta, 79 rue Manin, 75019 Paris.

15 Georges Perec, La Disparition, Paris, Denoël, 1969. Précisons à l'égard de ceux qui ne connaîtraient pas cet ouvrage, qu'il s'agit d'un roman de plus de trois cents pages pleines où ne s'emploie pas une seule fois la lettre "e". 
d'une écriture impersonnelle, formellement réglée et aussi lecturable et lisible que possible rompt catégoriquement avec le credo fondamental de l'épistémè littéraire de notre époque, qui continue à assimiler valeur littéraire et brisure expressive de tout carcan normatif. Ni spontanée, ni sauvage, ni sincère, la littérature hyperconstruite est absolument étrangère aux canons de l'esthétique contemporaine. Soucieuse des formes, utilisant activement les contraintes du discours, puisant volontiers dans les arcances de la rhétorique ancienne, elle paraît renouer avec les formes du passé, alors que c'est la relance d'une certaine modernité - interrompue par la surenchère facile des avant-gardes - qu'elle se donne pour tâche première. Dans cette perspective, sa marginalité est réelle, malgré le classicisme certain à hauteur de ses techniques.

\section{Les marges peu sûres de l'anachronisme}

Le problème que soulève le travail de l'hyperconstruction est beaucoup plus général que le problème de la relance de la seule modernité à une époque où le désir moderniste a cessé d'avoir cours. En effet, les difficultés qu'il pointe ont trait à l'évaluation correcte de l'anachronisme - vrai ou faux, c'est là justement le hic - à un moment historique où l'enchaînement linéaire des générations littéraires, chaque nouveau type d'écriture prenant la place ou le relais de l'autre au coeur du système, a perdu beaucoup de sa force d'explication. De ces glissements, l'impact sur la question de la marginalité devient de plus en plus visible. Car en même temps que se brouille la distinction entre les catégories de novateur et d'anachronique, la séparation du centre et des marges se fait plus évanescente.

S'il n'est pas directement poétique, l'exemple de Renaud Camus illustre fort bien les défaillances des anciennes antinomies, tant par les contenus et les formes de son oeuvre même que par la position "impossible" qu'elle occupe, et ce depuis un temps inhabituellement long, dans le système littéraire actuel. L'oeuvre même de Camus, au-delà d'une variété formelle et thématique qui n'a pas son égal ${ }^{16}$, pose à ses partisans comme à ses détracteurs d'épineux problèmes herméneutiques. Que penser en effet d'un auteur gay, chantre et militant de la cause homosexuelle (bien avant que cette "minorité" ne se soit évertuée à occuper son propre créneau

16 Sur Renaud Camus, cf. l'étude de J. Baetens Les mesures de l'excès, Paris, Les Impressions nouvelles, 1993. Pour une lecture parallèle mais plus circonscrit, consulter Richard-Laurent Barnett, "Sémiotique de l'excès" dans Reprises contemporaines: Colloque Lausanne, 1995. 
institutionnel), qui en même temps s'est fait le défenseur non moins acharné des idéaux que les homosexuels croient justement à la base de l'ordre social qui les opprime: la politesse, le savoir-vivre, la prééminence du paraître sur l'être, le goût et le soin des convenances, le jeu des formes? Faut-il en conclure que Renaud Camus sera réactionnaire pour les uns et progressiste pour les autres? Que sa position se caractérise par une ambivalence foncière? Pas vraiment. C'est en effet au nom même des valeurs qui lui ont permis de défendre l'homosexualité - comme choix et liberté face aux diktats d'une soi-disant nature marquée surtout par la violence et la répétition forcée de l'ordre ancien-, qu'il justifie l'éloge de valeurs et de principes trop rarement mis à contribution dans les discours minoritaires. La cohérence de ses prises de position démontre ainsi à quel point le jeu littéraire s'est structuré autour de critères commodes, mais trop simplistes pour pouvoir rendre compte de ce qui se passe réellement dans les textes. La place curieuse de cet auteur sur l'échiquier des lettres témoigne du reste de l'inconfort intellectuel durable qu'il provoque: cumulant des rôles de prime abord irréconciliables, Renaud Camus passe pour être un auteur à la fois obscur et illustre, archicommenté mais sans lecteurs, solitaire invétéré et porte-parole d'une époque entière.

Aussi est-ce - logiquement? - sur des problèmes de classification que débouche la réflexion sur la poétique des marges. Le cas de l'oeuvre camusienne est d'autant plus intéressant que l'auteur s'est beaucoup penché sur les questions de stratégie et de positionnement. Grâce au concept barthésien de barhmologie ou "science des degrés"17, dont il est le premier à tirer les effet savoureux qu'on sait, Renaud Camus permet de faire le départ entre les structures internes d'une écriture et le sens souvent tout autre qui lui est donné dans le spectre des interprétations possibles: "J'ai eu [. . . ] le sentiment que sur la plupart des points il n'y avait que deux positions possibles, mais qu'il importait de distinguer des niveaux à l'intérieur de chacune [.. .]"18. Un condamné à mort, par exemple, peut refuser ou accepter le bandeau qu'on lui offre face au peloton d'exécution, mais "il peut prendre l'une ou l'autre décision pour des raisons tout à fait différentes": courage, indifférence, snobisme, curiosité, etc. ${ }^{19}$

17 "Tout discours est pris dans le jeu des degrés. On peut appeler ce jeu: bathmologie, un néologisme n'est pas de trop, si l'on en vient à l'idée d'une science nouvelle: celle des échelonnements de langage. Cette science sera inouïe, car elle ébranlera les instances habituelles de l'expression, de la lecture et de l'écoute ('vérité', 'réalité', 'sincérité': son principe sera une secousse): elle enjambera, comme on saute une marche, toute expression", cf. Roland Barthes, Roland Barthes par Roland Barthes, Paris, Seuil, 1975, p. 71.

18 Renaud Camus, Buena Vista Park, Paris, Hachette, 1980, pp. 14-15.

19 Cet exemple est donné par Renaud Camus même, o.c. 
Pour l'écrivain, la forme la plus radicale de cette ouverture ou incertitude consiste bien sûr à semer le doute quant au sens même de son entreprise. Il arrive en effet que le projet se présente de telle façon qu'il n'est plus possible de voir s'il fait ou non encore partie de la littérature (c'est là un cas d'ironie suprême ${ }^{20}$, devant lequel beaucoup d'ironistes reculent à juste titre: ils pourraient bien y laisser des plumes, là où l'ironie autorise d'habitude à jouir tacitement d'une manière de supériorité). Il est aussi des auteurs qui écrivent mal à dessein - mais sans pouvoir le dire, bien sûr, tout aveu signifiant irrémédiablement l'échec de la manoeuvre ${ }^{21}$, de sorte que le public même averti ne sait plus comment juger une production pareille. Ainsi par exemple des vers de mirliton de Raymond Roussel, dont chaque nouvel alexandrin paraît "encore plus bête" que le précédent: signe de génie ou preuve de maladresse? Dans de quels cas, la question de la marginalité du discours ne se pose même plus: c'est le statut littéraire même de l'oeuvre qui laisse perplexe, ce qui en cas de réponse négative (plus d'un dira: "Ceci n'est pas de l'art", non que cela conteste leur vision traditionnelle de l'art, mais parce que cela échoue "piteusement" à en remplir les conditions) condamne le texte à un exil si radical que les notions classiques de cadre et de marge ne sont plus suffisantes.

Bref, pour peu que l'on traduise cette discussion en les termes de marginalitél centralité, on devrait admettre l'hypothèse que les textes les plus intéressants - les plus énigmatiques si l'on préfère - sont ceux où la charge d'indécidabilité est la plus intense; ceux qui se dérobent systématiquement à l'alternative proposée suffisamment marginaux pour être exclus du centre mais aussi suffisamment respectueux pour s'arroger le droit d'y rester. La vraie marginalité contemporaine, c'est dans ce non-lieu, cet impossible va-et-vient, cet entre-deux vide, qu'il importe de la chercher.

20 Jean-Benoît Puech, qui a bien étudié le phénomène, parle non sans raison de "suicide" littéraire, cf., Du vivant de l'auteur, Seyssel, Champ vallon, 1990. Pour plus de détails, voir RichardLaurent Barnett, "Des paroles qui se sont tues: prosopopée pour une marge défunte", Neophilologus (2000); et aussi, dans un tout autre contexte, "Le post-modernisme énigmatisé," dans Exégèses Nouvelles II, Bruxelles, 1992.

21 C'est le problème posé par la période "vache de Magritte", où le canular n'a pas tardé à se voir lourdement sougligné: le scandale provoqué par le "maître" surréaliste lorsqu'il s'est mis à faire des toiles "horribles", "commerciales", dans un but visiblement alimentaire, a vite perdu beaucoup de son impact à cause du clivage très net apparu entre les deux pans (artistique et non-artistique) de la production du peintre.

22 Cet essai de synthèse eût-il été réalisé sans le long entretien méditatif dont Julia Kristeva me fit l'amitié en 1999, à Paris? Trop rare privilège. 


\section{RESUMO}

Além e aquém dos a prioris e das convicções de qualquer um, o lque das margens constitui, em termos barthesianos, uma só e única "escritura", um só e único engajamento histórico em prol de um certo mode de inscrever seu discurso frente ao público e à história. Trata-se do reino exclusivo da atração pela margem, ou da rejeição do centro; trata-se também da estratégia sem falha do "dá licença, que agora chegou a minha vez". Trata-se por fim, da supremacia de um tipo de discurso no qual as qualidades do sujeito prevalecem, em toda parte, sobre a realização de seu enunciado.

Palavras-chave: Margem, marginalidade, desvio, paratexto, narratologia, critica contemporânea, texto-limite.

\section{RÉSUMÉ}

Au-delà et en-deça des a prioris et convictions, le choix des marges constitue, en termes barthésiens, une seule et même "écriture", un seul et même engagement historique d'une certaine façon d'inscrire sa parole face au public et face à l'histoire. C'est le règne sans partage du goût de la marge, ou du dégoût du centre; c'est aussi la stratégie sans faille de l"ôte-toi de là que je m"y mette". C'est enfin la domination d'un type de parole où les qualités du sujet prévalent partout sur l'achèvement de son énoncé.

Mots-clés: marge, marginalité, écart, paratexte, narratologie, critique contemporaine, texte-limite.

\section{BIBLIOGRAPHIE DES OUVRAGES CITÉS}

BAETENS, Jan. Les mesures de l'excès. Paris: Les Impressions Nouvelles, 1993.

L. L'éthique de la contrainte. Louvain: Peeters, 1995.

- Vérités du détour. Formules 1 (1997), 71-92.

BARNETT, Richard-Laurent. Des paroles qui se sont tues: prosopopée pour une marge défunte. In Neophilologus, 2000.

- Herméneutique et indicible. Travaux de Littérature (1999), 81-104.

—. Le post-modernisme énigmatisé. Exégèses Nouvelles II. Bruxelles, Presses de l'Université de Bruxelles, 1992. 
—. Poétique et marginalité. Seventeenth-Century French Studies (1998), 171 199.

—. Sémiotique de l'excès. Reprises contemporaines: colloque Lausanne, 1995.

- Sémiotique des marges. Paris: Seuil, 2000.

27-43.

- Transgressing Boundaries: The Poetics of Marginalisation. L'Esprit Créateur (1998),

BARTHES, Roland. Le degré zéro de l'écriture. Paris: Seuil, 1972.

- Roland Barthes par Roland Barthes. Paris, Seuil, 1975.

BORGES, Perspectivas modernas Madrid: University of Madrid, 1977).

CAMUS, Renaud. Buena Vista Park. Paris: Hachette, 1980.

COMPAGNON, Antoine. Les cinq paradoxes de la modernité. Paris: Seuil, 1990.

COSTE, Didier. Le retour d'Alexandre. Formules 1 (1997), 7-38.

DEBRAY, Régis. Vie et mort de l'image. Une histoire du regard en Occident. Paris: Gallimard, 1994.

ELZENSWEIG, Uri. Autopsies du roman policier. Paris: UGE - 10/18, 1983.

FALEMPIN, Michel. Une apparence de vie. Paris: Ivréa, 1995.

FISH, Stanley. Boutique Multiculturalism. Critical Inquiry (1997), 378-395.

PEETERS, Benoît. Les bijoux ravis. Bruxelles, Magic Strip, 1983.

PEREC, Georges. La disparition. Paris: Denoël, 1969.

PRIGENT, Christian. Anthologie TXT. Louvain: Bourgois, 1994.

- Ceux qui meredRent. Paris: P.O.L., 1991.

. Une erreur de la nature. Paris: P.O.L., 1996.

PUECH, Jean-Benoit. Du vivant de l'auteur. Seyssel: Champ vallon, 1990.

RICARDOU, Jean. Eléments de textique. Conséquénces (1996). 224-261.

ROUBAUD, Jacques. La boucle. Paris: Seuil, 1993.

—. Le grand incendie de Londres. Paris. Seuil, 1989.

. Mathématique. Paris: Seuil, 1996.

- La vieillesse d'Alexandre. Paris: Maspero, 1978. 